\title{
Bayesian inference and model choice for Holling's disc equation: a case study on an insect predator-prey system
}

\author{
N. E. Papanikolaou ${ }^{1,4}$, H. Williams², N. Demiris ${ }^{3}$, S. P. Preston ${ }^{2}$, P. G. Milonas ${ }^{1}$ \\ and T. Kypraios ${ }^{2}$ \\ ${ }^{1}$ Department of Entomology and Agricultural Zoology, Benaki Phytopathological Institute, St. Delta 8, Kifissia, 14561, \\ Greece \\ 2 School of Mathematical Sciences, University of Nottingham, University Park, NG7 2RD, UK \\ ${ }^{3}$ Department of Statistics, Athens University of Economics and Business, Patission 76, Athens, 10434, Greece \\ ${ }^{4}$ Corresponding author. E-mail: nepapanikolaou@yahoo.gr
}

Keywords: Bayes factors, Coccinellidae, Functional response, Markov Chain Monte Carlo, Ordinary differential equation models.

\begin{abstract}
The dynamics of predator-prey systems relate strongly to the density (in)dependent attributes of the predator's feeding rate, i.e., its functional response. The outcome of functional response models is often used in theoretical or applied ecology in order to extract information about the mechanisms associated with the feeding behavior of predators. The focus of this study centres upon Holling's type II functional response model, commonly known as the disc equation, which describes an inverse-density dependent mortality caused by a single predator to its prey. A common method to provide inference on functional response data involves nonlinear least squares optimization, assuming independent Gaussian errors, an assumption often violated in practice due to the heteroscedasticity which is typically present in the data. Moreover, as prey depletion is common in functional response experiments, the differential form of disc equation ought to be used in principle. We introduce a related statistical model and adopt a Bayesian approach for estimating parameters in ordinary differential equation models. In addition, we explore model uncertainty via Bayes factors. Our approach is illustrated via the analysis of several data sets concerning the functional response of a widespread ladybird beetle (Propylea quatuordecimpunctata) to its prey (Aphis fabae), predicting the efficiency of this predator on a common and important aphid species. The results showed that the approach developed in this study is towards a direction for accurate estimation of the parameters that determine the shape of the functional response of a predator without having to make unnecessary assumptions. The R (www.r-project.org) code for fitting the proposed model to experimental data is made freely available.
\end{abstract}

Abbreviations: MCMC-Markov Chain Monte Carlo, MLE-Maximum Likelihood Estimator, ODE-Ordinary Differential Equation, OLS-Ordinary Least Squares.

\section{Introduction}

The concept of functional response, a fundamental aspect of community ecology, describes the relationship between per capita predator consumption and prey density (Solomon 1949). Holling (1959a) proposed various types of functional response to provide a better understanding of the components of predator-prey interactions; namely, a linear (type I), a decelerating (type II), and a sigmoid (type III). In other words, the prey consumption is assumed to increase linearly with prey density or increase asymptotically to a plateau under type I and type II respectively, while in a type III functional response one assumes that the prey consumption is supposed to be of a sigmoid form (S-shaped) as prey density increases. Although more complex forms of the classical prey-dependent functional responses exist (see, for example Jeschke et al. 2002), a significant amount of interest has been drawn to Holling's type II and III functional responses because of their simplicity and tractability, balancing between reality and feasibility (see, for example Englund et al. 2011).
Holling's modelling approach for type II functional responses illustrates an inverse-density-dependent prey mortality model which is common among invertebrate predators (Hassell et al. 1977). Examining the workings of predator's individuals, Holling (1959b) developed a mechanistic model to explain their feeding behaviour, commonly known as the disc equation, which is an ordinary differential equation (ODE) of the form:

$$
\frac{d N(t)}{d t}=-\frac{a N}{1+a T_{h} N}
$$

where $N$ denotes the prey density, $a$ the predator's attack rate, i.e., the per capita prey mortality at low prey densities, and $T_{h}$ the handling time which reflects the time that a predator spends on pursuing, subduing, eating and digesting its prey. Despite its potentially simplified assumptions, a vast literature indicates that researchers often focus on the disc equation to describe predator's feeding behaviour, developing several concepts in ecology theory or modelling predator-prey dy- 
namics (see, for example Beddington 1975, Englund et al. 2011, Jeschke et al. 2002, Okuyama 2012a). Thus, it has become a baseline model in the sense of its determinant effect on much of modern ecology theory (Englund et al. 2011).

Given the importance of the disc equation on natural ecosystems, a number of early published papers investigated various statistical methods to infer the attack rate $(a)$ and the handling time $\left(T_{h}\right)$ from experimental data (see for example Fan and Petitt 1994, Livdahl 1979, Livdahl and Stiven 1983, Okuyama 2012b). One approach that has been commonly used is to linearize the disc equation to enable estimation of $a$ and $T_{h}$ within the framework of linear regression models. Linearizing a non-linear model, sometimes by making simplifying assumptions, is a method that has been attractive in the literature due to its ease of implementation. In this particular case, this can be done easily; setting $N=N_{0}$ on the right hand side of (1), where $N_{0}$ denotes the initial prey density, an analytic expression of $N(t)$ is available:

$$
N(t)=N_{0}-\frac{a N_{0} t}{1+a T_{h} N_{0}} .
$$

By rearranging and taking the reciprocals, one can derive expressions of the least square estimates of $a$ and $T_{h}$ explicitly (i.e., without numerical optimization). Nevertheless, such an approach relies on the assumption that the resource is not depleted during the experimental progress. Whilst there are cases in which that assumption is not unreasonable, such as in parasitoid-host systems, there are several cases where the resources are depleted over time; for instance, in predator-prey systems. Therefore, in such cases the differential form of the disc equation is to be preferred over its linear approximation, or the random predator equation (Rogers 1972) which is the integrated form of the disc equation.

Another approach to estimate the parameters of the disc equation given experimental data involves non-linear least squares optimization assuming identically and independently distributed (additive) Gaussian errors. However, such an assumption not only is likely to be violated by the heteroscedasticity which often arises in functional response data (Trexler et al. 1988), but this particular error distribution does not seem natural either, especially at early stages of the experiment where the number of prey consumed is low.

An interesting approach to modelling predation in functional response was developed by Fenlon and Faddy (2006) who studied two alternative model classes for such systems, one using likelihood-based inference for a beta-binomial model accounting for overdispersion and a counting-processbased framework. Although there are similarities to our basic modelling framework there are also important differences, namely we follow a distinct (Bayesian) approach to inference and model selection and our computational framework does not resort to asymptotic normality. In addition, our model differs in the way it accounts for density dependence.

The main aim of this paper is to introduce a hierarchical model which in principle can incorporate any of Holling's various types of functional response and accounts for heteroscedasticity. Also, in spite of numerical differential equation solvers making it perfectly feasible to use richer ODE models, there is still a tendency for researchers to use simpler models on grounds of convenience (e.g., the random predator equation). Therefore, we aimed to show that it is perfectly feasible to work with the richer models, providing clear statistical evidence of the benefit of doing so. In addition, we illustrate how one can estimate the parameters of this model within a Bayesian framework and select between competing models (hypotheses) given experimental data. The proposed model and methodology are illustrated via the analysis of eight data sets which involve the functional response of a predatory insect to its prey. In particular, the ladybird beetle Propylea quatuordecimpunctata L. (Coleoptera: Coccinellidae) and its essential prey Aphis fabae Scopoli (Hemiptera: Aphididae) were used as case study organisms. Aphis fabae is well recognized as a serious pest of cultivated plants worldwide (Blackman and Eastop 2000), where P. quatuordecimpunctata is a widely distributed aphidophagous coccinellid (Hodek et al. 2012). As a thoroughly estimating of biological control agents' functional response is of importance, with this application we provide a quantified analysis of the intake rate of $P$. quatuordecimpunctata as a function of $A$. fabae density.

\section{Materials and methods}

\section{Data collection and experimental conditions}

An A. fabae colony originated from a stock colony at the Biological Control Laboratory, Benaki Phytopathological Institute, reared on Vicia faba L. plants at $20 \pm 1{ }^{\circ} \mathrm{C}, 65 \pm 2 \%$ RH and a photoperiod of 16:8 L:D. Propylea quatuordecimpunctata was collected from Zea mays L. plants infested with Rhopalosiphum maidis Fitch in Arta County (Northwestern Greece). The coccinellid was reared in large cylindrical Plexiglass cages (50 cm length $30 \mathrm{~cm}$ diameter) containing $A$. fabae prey on potted $V$. faba plants at $25 \pm 1{ }^{\circ} \mathrm{C}, 65 \pm 2 \% \mathrm{RH}$ and a photoperiod of 16:8 L:D. The experiments were carried out at $20 \pm 1{ }^{\circ} \mathrm{C}, 65 \pm 2 \% \mathrm{RH}$ and a photoperiod of $16: 8$ L:D. The experimental arena consisted of a plastic container (12 cm height $\times 7 \mathrm{~cm}$ diameter) with a potted $V$. faba plant host (at 8-9 cm height, top growth was cut) with different $A$. fabae densities (3-3.5 days-old). An individual larva, female or male of $P$. quatuordecimpunctata was placed into plastic containers, having starved for $12 \mathrm{~h}$. Total exposure time of prey and predator was $24 \mathrm{~h}$. Aphis fabae densities were 2, 4, 8, 16 and 32 aphids for $1^{\text {st }}$ instar larvae, 2, 4, 8, 16, 32 and 64 aphids for $2^{\text {nd }}$ instar larvae, 4, 8, 16, 32, 64 and 128 aphids for $3^{\text {rd }}$ and $4^{\text {th }}$ instar larvae as well as female and male adults. We used 20-30 day old P. quatuordecimpunctata adults. Ten replicates of each prey density were formed. Functional response experiments were also run at $25 \pm 1{ }^{\circ} \mathrm{C}$ for female and male adults. The data sets concerning the functional response of larvae were used in a previous study of Papanikolaou et al. (2011). 


\section{A hierarchical model}

Denote by $N_{e}(t)$ the number of prey eaten by time $t$. Since a prey item is either dead or alive by time $t$ (which often denotes the end of the experiment), we assume that $N_{e}(t)$ follows a Binomial distribution with parameters $N_{0}$ and $p(t)$, where $N_{0}$ is the initial prey population and $p(t)$ is the probability that a prey item has been eaten by time $t$ :

$$
\begin{array}{r}
N_{e}(t) \sim \operatorname{Binom}\left(N_{0}, p(t)\right) \\
p\left(t / a, T_{h}\right)=\left(N_{0}-N\left(t / a, T_{h}\right)\right) / N_{0}
\end{array}
$$

where $N(t)$ is given by the solution of the ordinary differential equation (1) and evaluated at time $t$. Notice that (1) cannot be solved analytically and hence the solution has to be derived numerically. Furthermore, in principle any functional response model for $N(t)$ can be used and not just the model as given in (1)

\section{Bayesian inference}

Preliminaries. Traditionally, parameter estimation for models concerned with functional response has been done by searching for the set of parameters (i.e., attack rate, handling time, etc) for which the model and data match most closely according to some criterion, such as the sum of squared differences. Such an ordinary least squares (OLS) approach provides an estimate for the parameter values that gives the "best fit" to the experimental data, but it gives no information about uncertainty in the estimate; for example, whether or not there are other plausible values of parameters that also give equally good fits. Thus, being able to quantify the uncertainty surrounding the ability of our point estimates to reflect the (unknown) truth is an equally important aspect in parameter estimation. Typically, researchers resort to normality assumptions whence OLS coincide with the maximum likelihood estimators (MLEs), leading to quantification of the uncertainty around the MLEs.

In this paper, we adopt the Bayesian paradigm which enables us to quantify the uncertainty of our estimates in a coherent, probabilistic manner (e.g., Bolker 2008). We utilise a Markov Chain Monte Carlo (MCMC) algorithm (see, for example, Brooks et al. 2011, Gelman et al. 1996) to sample from the posterior density of the parameters of interest

Prior distributions. We assume little prior knowledge of the attack rate $(a)$ and handling time $\left(T_{h}\right)$ when making inference for the parameters of our model. In particular, we assume that both of them have independent slowly varying Exponential distributions:

$$
\begin{aligned}
& a \sim \operatorname{Exp}\left(\lambda_{1}\right) \\
& T_{h} \sim \operatorname{Exp}\left(\lambda_{2}\right)
\end{aligned}
$$

and we typically set $\lambda_{1}$ and $\lambda_{2}$ to $10^{-6}$ in order to achieve large prior variance. Assigning Exponential distribution with low rates is a typical choice when one is interested in assuming a non-informative distribution about the parameters. In other words, our prior belief is expressed via a practically flat den- sity over realistically plausible positive real numbers (e.g., between 0 and 100), allowing for the data to mostly inform the posterior density of $a$ and $T_{h}$. We have used non-informative priors for the attack rate and the handling time. Although the maximum likelihood estimates will coincide with the maximum a posteriori probability estimates in this case, we advocate the use of a Bayesian approach since, in principle, one can assign informative priors to either parameter (e.g., using information from past experiments) and most importantly, offers a particularly natural way to select between candidate models.

Likelihood. We now derive the likelihood of the observed data under the proposed hierarchical model. Given that all the experiments lasted for 24 hours and for the ease of exposition, we drop the dependence of $t$ in the notation. Denote by $X=\left\{\left(x_{i}, n_{i}\right)_{k}\right\}, i=1, \ldots, m$ and $k=1, \ldots, K$ the observed data of a functional response experiment; the index $i$ refers to the different initial prey densities that were used in the experiment and $k$ refers to each replication. Essentially, the observed data consist of pairs of initial prey density and number of prey eaten after 24 hours.

An observed dataset is presented in Table 1 for illustration; the second column $\left(n_{j}\right)$ consists of the initial prey densities for $j=1, \ldots, 6$ and the rest, $\left(x_{1}, \ldots, x_{j}\right)$ refer to the number of prey eaten by the predator after 24 hours.

The probability of observing $x_{j}$ prey items eaten out of $n_{j}$ prey after 24 hours is given by:

$$
P\left(x_{j}=x / n_{j}(0), a, T_{h}\right)=\left(\begin{array}{l}
n_{j} \\
x
\end{array}\right) p^{x}(1-p)^{n_{j}-x},
$$

where $p=P\left(t / a, T_{h}\right)$ is given in Equation 2 for $t=24$ and therefore is implicitly dependent upon $a$ and $T_{h}$ via (1). Assuming independence between the $k$ replicates in each experiment as well as between the different experiments, the likelihood of the observed data $X$ given the parameters $\theta=\left(a, T_{h}\right)$ after $T=$ 24 hours is written as follows:

$$
L\left(a, T_{h}\right)=\pi(X / a, \theta)=\prod_{\kappa} \prod_{j} P\left(x_{j} / n_{j}, a, T_{h}\right) .
$$

Posterior distribution. Equations 3, 4 and 6 give rise to the posterior distribution whose density is given as follows:

$$
\begin{aligned}
& \pi\left(a, T_{h} / X\right) \propto \prod_{k} \prod_{j} P\left(x_{j} / n_{j}, a, T_{h}\right) \times \\
& \times \lambda_{1} \lambda_{2} \exp \left\{-\lambda_{1} a-\lambda_{2} T_{h}\right\}
\end{aligned}
$$

The posterior density of interest (Equation 7) is not of a closed form due to its normalising constant not being available explicitly. Therefore, in this study we employed to a random walk Metropolis algorithm (Metropolis et al. 1953, Gelman et al. 1997, Gamerman and Lopes 2006) to draw samples from $\pi\left(a, T_{h} / X\right)$. 
Table 1. Number of prey items consumed by Propylea quatuordecimpunctata male adults for each trial $(i=1, \ldots, 10)$. The experiment was conducted at $20^{\circ} \mathrm{C}$ for six different Aphis fabae prey densities $\left(n_{i j}, j=1, \ldots, 6\right)$. Therefore, $x_{i j}$ denotes the count of consumed prey at the $j$ th density at the $i$ th trial.

\begin{tabular}{cccccccccccc}
\hline$j$ & $n_{j}$ & $x_{1 j}$ & $x_{2 j}$ & $x_{3 j}$ & $x_{4 j}$ & $x_{5 j}$ & $x_{6 j}$ & $x_{7 j}$ & $x_{8 j}$ & $x_{9 j}$ & $x_{10 j}$ \\
\hline 1 & 4 & 4 & 4 & 4 & 4 & 4 & 4 & 4 & 4 & 4 & 4 \\
2 & 8 & 8 & 8 & 8 & 8 & 8 & 2 & 8 & 8 & 7 & 7 \\
3 & 16 & 8 & 14 & 10 & 10 & 15 & 14 & 12 & 14 & 9 & 16 \\
4 & 32 & 16 & 27 & 18 & 16 & 23 & 20 & 17 & 21 & 31 & 17 \\
5 & 64 & 30 & 29 & 33 & 24 & 30 & 22 & 20 & 26 & 26 & 27 \\
6 & 128 & 50 & 36 & 28 & 26 & 24 & 41 & 30 & 38 & 28 & 42 \\
\hline
\end{tabular}

Bayesian model choice. Statistical inference, in general, is not limited to parameter estimation. Another common goal is hypothesis testing, in which we are interested in discriminating models in order to gain a better understanding of the structure of the statistical model(s) of interest and facilitate for model-robust decision making. Here we are interested in observing the extent to which the observed data support the scientific hypothesis that the differential form of the disc equation is to be used when prey is depleted during the functional response experiments.

Bayes factors. The Bayesian approach to model selection (or discrimination) is based upon an extension to the posterior distribution to include not only uncertainty regarding the model parameters but also for the model itself. Consider the following framework: suppose we observe data $X$ and have a series of plausible models indexed by $w=1, \ldots, W$. Denote by $\theta_{w}$ the vector of parameters associated with model $M_{w}$ and by $\pi_{w}\left(X / \theta_{w}\right)$ the likelihood of the observed data under model $W$. Then by specifying a prior distribution $p_{k}\left(\theta_{w}\right)$ for the model parameters under each model and a prior probability for each model, $p\left(M_{w}\right)$, we can derive the joint posterior distribution over both the model and parameter spaces, given by

$$
\pi\left(\theta_{w}, M_{w} / X\right) \propto \pi_{w}\left(X / \theta_{w}\right) \pi_{w}\left(\theta_{w}\right) \pi\left(M_{w}\right)
$$

Assuming prior independence between $M_{w}$ and $\theta_{w}$, the joint posterior distribution can then be written down (using Bayes Theorem) as product of two components:

$$
\pi\left(\theta_{w}, w / X\right)=\pi\left(\theta_{w} / w, X\right) \pi(w / X)
$$

where $\pi\left(\theta_{w}, M_{w} / X\right)$ is the posterior distribution of the parameters under model $M_{w}$ and $\pi\left(M_{w} / X\right)$ denotes what we refer to as the "posterior model probability" which represents our beliefs, after observing data $X$, of what is the chance that model $M_{w}$ is the true model given that one of models $1, \ldots, W$ is true.

Once these posterior model probabilities are obtained they can then be used to discriminate between the competing models by computing the Bayes Factor which is simply defined as the ratio of the posterior odds, i.e., the ratio of the posterior to the prior model probability:
$B F_{12}=\frac{\pi\left(X / M_{w}=1\right) / \pi\left(M_{w}=1\right)}{\pi\left(X / M_{w}=2\right) / \pi\left(M_{w}=2\right)}$

In other words rearranging Equation 10 shows that posterior odds $=$ Bayes factor $\times$ prior odds.

The value of the Bayes factor represents the relative likelihood of $M_{1}$ to $M_{2}$ and is of practical appeal because its value is independent of the choice of the prior model probabilities (see Kass and Raftery 1995). It is easy to see that when the models are equally probable a priori so that $\pi\left(M_{w}=1\right)=$ $\pi\left(M_{w}=2\right)=0.5$ the Bayes factor is equal to the posterior odds in favour of $M_{1}$. The quantity $\pi\left(X / M_{w}\right)$ for $k=1,2$ in (10) is obtained by integrating over the parameter space,

$\pi\left(X / M_{w}\right)=\int_{\theta_{w}} \pi\left(X / \theta_{w}, M_{w}\right) \pi_{w}\left(\theta_{w}\right) d \theta_{w}$

where $\theta_{w}$ is the parameter vector under model $M_{w}$ and $\pi_{w+}\left(\theta_{w}\right)$ is its prior density. The term $\pi\left(X / M_{w}\right)$ is the marginal probability of the data and is often called the marginal or integrated likelihood in the statistical literature while it is typically referred to as the evidence in the physics and machine learning communities. The Bayes factor is, therefore, a summary of the evidence provided by the data in favour of one hypothesis represented by a statistical model as opposed to another. Note that this formulation is completely general and does not require nested models, as is typically the case with likelihood ratio tests. Additionally, no asymptotic justification is required so that these results can be used for moderate sample sizes as well.

The marginal likelihoods are rarely available in analytic form. Therefore, in practice if the number of parameters in each model is not very large (typically 2-5 parameters), then the marginal likelihoods and consequently the Bayes factors are obtained via straightforward numerical integration. However, if the dimension of the parameter vector $\theta_{\mathrm{w}}$ is very large then computational tools such as trans dimensional MCMC algorithms (Green 1995) can be used instead to explore the more complex posterior distribution described above. 
Table 2. Parameter values of Holling's disc equation obtained as posterior means ( $95 \%$ Credible Intervals), and the correlation of attack rate and handling time $(95 \%$ Credible Intervals).

\begin{tabular}{llll}
\hline & attack rate & handling time & correlation \\
\hline \multirow{2}{*}{$1^{\text {st } \text { instar }}$} & 0.1496 & 7.1195 & 0.2393 \\
& $(0.0728-0.2578)$ & $(5.6887-8.7337)$ & $(0.2197-0.2587)$ \\
$2^{\text {nd } \text { instar }}$ & 0.1324 & 2.6713 & 0.1035 \\
& $(0.0976-0.1763)$ & $(2.3351-3.0357)$ & $(0.0830-0.1239)$ \\
3 rd instar & 0.1514 & 1.1567 & 0.0453 \\
& $(0.1230-0.1864)$ & $(1.0596-1.2605)$ & $(0.0246-0.0659)$ \\
$4^{\text {th instar }}$ & 0.2025 & 0.5215 & 0.0864 \\
& $(0.1744-0.2373)$ & $(0.4865-0.5575)$ & $(0.0659-0.1069)$ \\
females $\left(20^{\circ} \mathrm{C}\right)$ & 0.2278 & 0.5058 & 0.0728 \\
& $(0.1898-0.2737)$ & $(0.4071-0.6273)$ & $(0.0523-0.0934)$ \\
males $\left(20^{\circ} \mathrm{C}\right)$ & 0.1067 & 0.6507 & 0.1600 \\
& $(0.0889-0.1265)$ & $(0.5143-0.7735)$ & $(0.1396-0.1798)$ \\
females $\left(25^{\circ} \mathrm{C}\right)$ & 0.2193 & 0.2565 & 0.1538 \\
males $\left(25^{\circ} \mathrm{C}\right)$ & $(0.1910-0.2494)$ & $(0.2237-0.2881)$ & $(0.1335-0.1740)$ \\
& 0.1970 & 0.4805 & 0.1994 \\
& $(0.1666-0.2321)$ & $(0.4104-0.5608)$ & $(0.1795-0.2192)$ \\
\hline
\end{tabular}

\section{Results and discussion}

The functional response is a fundamental characteristic of predator-prey systems. We have developed a hierarchical model which accounts for heteroscedasticity and illustrated how to infer the parameters of interest (e.g., the attack rate and the handling time) within a Bayesian framework using MCMC methods. In addition, we showed how one can assess competing scientific hypotheses by investigating which model is mostly supported by the experimental data. Generally, ODEs are frequently used in representing consumer-resource interactions and the outcome of such models is therefore of great interest to researchers. Thus, we have made our computer code implementing the present analysis in $\mathrm{R}$ ( $\mathrm{R}$ Core team 2013) publicly available on http://www.maths.nott.ac.uk/ tk/ files/functional_response/, to encourage and allow researchers to fit (and compare) the proposed models to their datasets.

In practice, we often summarize the posterior distribution of the parameters by calculating a variety of interpretable summary statistics such as posterior means, medians and credible intervals. The posterior means of both parameters of the disc equation obtained are presented in Table 2. By inspecting the $95 \%$ credible intervals we observe that the estimated attack rates were similar for all four larval stages of the predator, indicating that the larvae have similar abilities to respond to increasing prey densities. On the other hand, handling times decreased for the older larvae. This further indicates an increase in the upper level of the response, leading older larvae to a higher consumption of prey. Being larger gives them an advantage in handling prey. At $20^{\circ} \mathrm{C}$, the attack rate for females was higher than those for males. This means that at low prey densities (i.e., at the supplied prey densities that the predator is not satiated) the females have the ability to consume more prey items than the males. However, comparison of handling times yielded no differences, indicating that both sexes have similar maximum predation ability. Overall, at $20{ }^{\circ} \mathrm{C}$ we expect that females, males and fourth instar larvae of $P$. quatuordecimpunctata to display the higher predation ability among predators stages. This could be of great interest for biological control practitioners, since these stages are to be preferred in potential release of this predator in agroecosystems, allowing an influential decrease of aphid pests.

Our results also showed that at the temperature of $25^{\circ} \mathrm{C}$ there was a notable difference of estimated handling times between males and females. This further indicates that females might prey and subdue prey more efficiently and faster than males. Moreover, handling time increased considerably as temperature decreased from $25{ }^{\circ} \mathrm{C}$ to $20{ }^{\circ} \mathrm{C}$ for females, but not for males. According to Papanikolaou et al. (2013), the fecundity of $P$. quatuordecimpunctata females is higher at $25^{\circ} \mathrm{C}$ than $20^{\circ} \mathrm{C}$, where females of roughly $20-30$ day-old exhibit their maximum reproductive potential at $25{ }^{\circ} \mathrm{C}$. As a consequence, higher energy requirements for egg production lead them to higher consumption of prey. Additionally, attack rate for males was lower at $20^{\circ} \mathrm{C}$ than $25^{\circ} \mathrm{C}$ unlike females, as it was not different among these temperatures. Attack rate might follow a hump-shaped relationship with temperature as it happens for the ladybird Coleomegilla maculata lengi DeGeer (Sentis et al. 2012). The two temperatures examined here might have been at the plateau of the hump-shaped relationship with temperature for females and therefore no differences occurred, whereas, for males was still increasing with temperature.

Although investigating the Pearson's correlation between the estimated parameters of the disc equation appears to be mostly ignored in the ecological literature, it is important to do so since this may reveal potential parameter non-identifiability issues as well as biological insights. Table 2 reveals a moderate but statistically significant positive correlation between the estimated handling times and the estimated attack rates of the predator, based on $95 \%$ credible intervals. This is biologically intuitive since coccinellids are being highly 
Table 3. The Bayes factor of $M_{2}$ versus $M_{1}$ for different values of the prior's hyperparameter $\lambda$.

\begin{tabular}{lllll}
\hline & $\lambda=0.01$ & $\lambda=0.1$ & $\lambda=1$ & $\lambda=10$ \\
\hline $1^{\text {st } i n s t a r}$ & 8.17 & 7.61 & 4.04 & 0.27 \\
$2^{\text {nd }}$ instar & 175.91 & 170.71 & 151.21 & 8.50 \\
$3^{\text {rd }}$ instar & $1.65 \times 10^{7}$ & $1.62 \times 10^{7}$ & $1.31 \times 10^{7}$ & $1.64 \times 10^{6}$ \\
$4^{\text {th }}$ instar & $1.09 \times 10^{21}$ & $1.06 \times 10^{21}$ & $8.50 \times 10^{20}$ & $9.21 \times 10^{19}$ \\
females $\left(20^{\circ} \mathrm{C}\right)$ & $1.32 \times 10^{20}$ & $1.29 \times 10^{20}$ & $9.98 \times 10^{19}$ & $7.95 \times 10^{18}$ \\
males $\left(20^{\circ} \mathrm{C}\right)$ & 0.12 & 0.12 & 0.11 & 0.04 \\
females $\left(25^{\circ} \mathrm{C}\right)$ & $7.56 \times 10^{22}$ & $7.41 \times 10^{22}$ & $6.00 \times 10^{22}$ & $7.81 \times 10^{21}$ \\
males $\left(25^{\circ} \mathrm{C}\right)$ & $5.60 \times 10^{14}$ & $5.49 \times 10^{14}$ & $4.50 \times 10^{14}$ & $5.56 \times 10^{13}$ \\
\hline
\end{tabular}

Table 4. The Bayes factor of $M_{2}$ versus $M_{3}$ for different values of the prior's hyperparameter $\lambda$.

\begin{tabular}{lllll}
\hline & $\lambda=0.01$ & $\lambda=0.1$ & $\lambda=1$ & $\lambda=10$ \\
\hline $1^{\text {st }}$ instar & 1.47 & 1.53 & 2.08 & 1.55 \\
$2^{\text {nd }}$ instar & 29.83 & 29.93 & 30.67 & 20.91 \\
$3^{\text {rd } i n s t a r ~}$ & 56.41 & 55.56 & 47.78 & 10.70 \\
$4^{\text {th }}$ instar & 1.69 & 1.67 & 1.43 & 0.30 \\
females $\left(20^{\circ} \mathrm{C}\right)$ & 132614 & 130522 & 111268 & 22586 \\
males $\left(20^{\circ} \mathrm{C}\right)$ & $2.64 \times 10^{14}$ & $2.61 \times 10^{14}$ & $2.33 \times 10^{14}$ & $7.38 \times 10^{13}$ \\
females $\left(25^{\circ} \mathrm{C}\right)$ & 614829 & 604344 & 508693 & 91812 \\
males $\left(25^{\circ} \mathrm{C}\right)$ & 6309 & 6230 & 5497 & 1559 \\
\hline
\end{tabular}

voracious, especially larvae which consume more prey items than they need for their development (Hodek et al. 2012). This trend may lead to a gradual increase of the handling time, as the attack rate increases.

In a previous study (Papanikolaou et. al. 2011) the authors fitted the non-differential form of the disc equation using a non-linear least squares approach, in order to provide inference for the functional response of $P$. quatuordecimpunctata larvae. The values of attack rates are notably lower than those estimated in the present analysis, indicating that linearisation may induce estimation bias. The attack rate coefficient illustrates the per capita prey consumption at low prey densities, indicating the initial slope of the functional response curve. A biased estimate of this parameter leads to underestimation of prey consumption at the lower prey densities, in which the handling time is not the limiting factor of the predation. In addition, a high value of the attack rate coefficient shows that the predator may exhibit stronger density-dependent predation behavior. In contrary, the values of the larvae handling times are close to those estimated in the present analysis. Handling time depicts a more complex behavior which includes a number of distinguish predator activities, such as pursuing, subduing, eating and digesting a prey item.

$$
\begin{array}{ll}
M_{1}: & \frac{d N(t)}{d t}=-\frac{a N_{0}}{1+a T_{h} N_{0}} \\
M_{2}: & \frac{d N(t)}{d t}=-\frac{a N}{1+a T_{h} N}
\end{array}
$$

\section{Model selection}

We applied the proposed method in two cases:

a) Our hypothesis is translated into two different models, describing type II functional responses; in particular

Note that the model $M_{1}$ uses the functional response used Papanikolaou et al. (2011) while $M_{2}$ uses the hierarchical

$$
\begin{aligned}
& M_{2}: \quad \frac{d N(t)}{d t}=-\frac{a N}{1+a T_{h} N} \\
& M_{3}: \frac{d N(t)}{d t}=-\frac{a N^{2}}{1+a T_{h} N^{2}},
\end{aligned}
$$

model that is proposed in Material and Methods.

b) In this case, our aim was to distinguish between type II and type III functional responses, which is of importance in functional response studies (Juliano 2001), i.e., where the model $M_{3}$ describes type III functional responses.

In each case, we assumed that both models are equally likely a priori and consider Exponential prior distributions for both parameters, $a \operatorname{Exp}(\lambda), T_{h} \operatorname{Exp}(\lambda)$. It is well known that the Bayes factor can be sensitive to the choice of model parameter's prior distributions. Therefore, we computed the Bayes factor for a range of different values of $\lambda$, namely, 0.01 , $0.1,1$, and 10 . We first computed the log of the marginal likelihoods for both models via numerical integration and then the Bayes Factors of model $M_{2}$ versus $M_{1}$ in the first case and $M_{2}$ versus $M_{3}$ in the second case. Tables 3 and 4 show the Bayes Factors of model $M_{2}$ versus model $M_{1}$ and $M_{2}$ versus 
$M_{3}$, respectively, for the different datasets and for different prior distributions. It is immediately apparent that $M_{1}$ is to be preferred in all but one cases (males at $20{ }^{\circ} \mathrm{C} M_{1}$ is to be preferred). Furthermore, the conclusions appear to be robust to the different choice of $\lambda$.

Type II functional responses are frequent in nature, especially among aphidophagous ladybirds (Hodek et al. 2012) and are typically described by Holling's disc equation, one of the most commonly used models in ecology. Our study allowed us to predict the efficiency of $P$. quatuordecimpunctata on a common and important aphid species. Since biological control practitioners often rely on functional response studies to design and use efficiently biocontrol agents, an accurate and non-biased estimation of the functional response parameters is of crucial importance. The approach developed here is towards that direction, for a more precise estimation of the parameters that determine the shape of the functional response of a predator. Also, functional response parameters of $P$. quatuordecimpunctata preying on A. fabae may be incorporated in predator-prey models evaluating the population dynamics of the study organisms.

From a statistical viewpoint routine Bayesian inference and model selection for ODE-based models remains a challenge for a number of reasons which relate to the need for solving the ODEs numerically. With respect to the former one may extend our methods by utilising gradient-based information for the construction of efficient MCMC proposals. The issue of model selection can be further explored by methodology based upon thermodynamic integration (Friel and Pettitt 2008). Such an approach is appealing in cases where numerical integration might be infeasible due to the large number of parameters in the model, resulting in the evaluation of highdimensional integrals. These are important directions for future research.

\section{References}

Beddington J. 1975. Mutual interference between parasites or predators and its effect on searching efficiency. J. Anim. Ecol. 44(1):331-340.

Blackman, R.L. and V.F. Eastop. 2000. Aphids on the World's Crops. An Identification and Information Guide. John Wiley \& Sons, Chichester

Bolker, B. 2008. Ecological Models and Data in R. Princeton University Press.

Brooks, S., A. Gelman, G. Jones and X.L. Meng. 2011. Handbook of Markov Chain Monte Carlo. Taylor \& Francis, Boca Raton.

Englund, G., G. Ohlund, C.L. Hein and S. Diehl. 2011. Temperature dependence of the functional response. Ecol. Lett. 14(9):914921.

Fan, Y. and F.L. Petitt. 1994. Parameter estimation of the functional response. Environ. Entomol. 23(4):785-794.

Fenlon, J.S. and Faddy M.J. 2006. Modelling predation in functional response Ecol. Model. 198: 154-162.

Friel, N. and A.N. Pettitt. 2008. Marginal likelihood estimation via power posteriors. J. R. Stat. Soc. B 70(3):589-607.

Gamerman, D. and H.F. Lopes. 2006. Markov Chain Monte Carlo: Stochastic Simulation for Bayesian Inference. Chapman and Hall/CRC, Boca Raton.
Gelman, A., G. Roberts and W. Gilks. 1996. Efficient metropolis jumping rules. In Bernado, J.M. et al. (eds), Bayesian Statistics, volume 5, page 599. Oxford Univ. Press, Oxford.

Gelman, A., W.R. Gilks and G. Roberts. 1997. Weak convergence and optimal scaling of random walk Metropolis algorithms. Ann. Appl. Probab. 7(1):110-120.

Green, P.J. 1995. Reversible jump Markov chain Monte Carlo computation and Bayesian model determination. Biometrika 82(4):711-732.

Hassell, M., J. Lawton and J. Beddington. 1977. Sigmoid functional responses by invertebrate predators and parasitoids. J. Anim. Ecol. 46(1): 249-262.

Hastings, W. 1970. Monte Carlo samping methods using Markoy chains and their applications. Biometrika 57: 97-109.

Hodek, I., H.F. van Emden and A. Honěk. 2012. Ecology and Behaviour of the Ladybird Beetles (Coccinellidae). WileyBlackwell, Chichester.

Holling, C.S. 1959a. The components of predation as revealed by a study of small-mammal predation of the European pine sawfly. Can. Entomol. 91:293-320.

Holling, C.S. 1959b. Some characteristics of simple types of predation and parasitism. Can. Entomol. 91:385-398.

Jeschke, J.M., M. Kopp and R. Tollrian. 2002. Predator functional responses: discriminating between handling and digesting prey. Ecol. Monogr. 72(1):95-112.

Juliano, S.A. 2001. Nonlinear curve fitting: predation and functional response curves. In: S.M. Scheiner and J. Gurevitch (eds), Design and Analysis of Ecological Experiments. Oxford University Press, Oxford, UK, pp. 178-196.

Kass, R.E. and A.E. Raftery. 1995. Bayes factors. J. Am. Stat. Ass. 90: 773-795.

Livdahl, T.P. 1979. Evolution of handling time: the functional response of a predator to the 450 density of sympatric and allopatric strains of prey. Evolution 33(2):765-768

Livdahl, T.P. and A.E. Stiven. 1983. Statistical difficulties in the analysis of predator functional response data. Can. Entomol. 115:1365-1370.

Metropolis, N., A.W. Rosenbluth, M.N. Rosenbluth, A.H. Teller and E. Teller. 1953. Equation of state calculations by fast computing machines. J. Chem. Phys. 21:1087-1092.

Okuyama, T. 2012a. Flexible components of functional responses. $J$. Anim. Ecol. 81:185-189.

Okuyama, T. 2012b. A likelihood approach for functional response models. Biol. Contr. 60(2):103-107.

Papanikolaou, N.E., P.G. Milonas, D.C. Kontodimas, N. Demiris and Y.G. Matsinos. 2013. Temperature-dependent development, survival, longevity and fecundity of Propylea quatuordecimpunctata (Coleoptera: Coccinellidae). Ann. Entomol. Soc. Am. 106(2):228-234.

Papanikolaou, N.E., A.F. Martinou, D.C. Kontodimas, Y.G. Matsinos and P.G. Milonas. 2011. Functional responses of immature stages of Propylea quatuordecimpunctata (Coleoptera: Coccinellidae) to Aphis fabae (Hemiptera: Aphididae). Eur. J. Entomol. 108(3):391-395.

R Core Team. 2013. R: A Language and Environment for Statistical Computing. R Foundation for Statistical Computing, Vienna, Austria. https://www.R-project.org/.

Rogers, D.J. 1972. Random search and insect population models. $J$. Anim. Ecol. 41: 369-383.

Sentis, A., J.L. Hemptinne and J. Brodeur. 2012. Using functional response modeling to investigate the effect of temperature 
on predator feeding rate and energetic efficiency. Oecologia 169(4):1117-1125.

Solomon, M. 1949. The natural control of animal populations. $J$. Anim. Ecol. 18(1):1-35.
Trexler, J., C. McCulloch and J. Travis. 1988. How can the functional response best be determined? Oecologia 76:206-214.

Received November 9, 2015 Revised May 17, 2016 Accepted May 25, 2016 